\title{
Movement of interstitial clusters in stress gradients of grain boundaries
}

\author{
M. Samaras, P. M. Derlet, and H. Van Swygenhoven \\ Paul Scherrer Institute, 5232 Villigen, Switzerland \\ M. Victoria \\ CRPP-Fusion Technology Materials, EPFL, CH-5232 Villigen-PSI, Switzerland
}

(Received 25 June 2003; published 30 December 2003)

\begin{abstract}
Simulations of nanocrystalline materials reveal that the pressure gradient present within the structure can play a key role in the movement of self-interstitial atoms (SIA's) to surrounding grain boundaries and therefore in the resulting defect structure formed during displacement cascades. Initially SIA's sense the grain boundary region as a "defect collector plate," a two-dimensional (2D) indistinguishable region under tension to which they are attracted. The SIA's approach the "defect collector plate" and at a certain distance are able to distinguish the local variations in pressure specific to the particular grain boundary misorientation, changing their direction in response to the local pressure environment. Consequently even large SIA clusters undergo a change in direction, moving $1 \mathrm{D} / 3 \mathrm{D}$ in order to reach and follow a lower compressive and where possible a tensile pressure path to the grain boundary.
\end{abstract}

DOI: 10.1103/PhysRevB.68.224111

PACS number(s): $61.80 . \mathrm{Hg}, 81.07 . \mathrm{Bc}, 02.70 . \mathrm{Ns}$

\section{INTRODUCTION}

Molecular dynamics (MD) computer simulations of displacement cascade evolution has provided a better understanding of the atomistic behavior of materials during the initial stages of radiation damage. Simulations have been performed predominately in single crystal (for a review see Ref. 1) and recently in bicrystal ${ }^{2,3}$ and nanocrystalline $(n c)^{4-7}$ samples. The differences in final defect structure of the various samples show that grain boundaries (GB's) play an important role in the evolution of the cascade. In the context of neutron and proton irradiation of materials, a GB has long been considered as a sink for both self-interstitial atoms (SIA's) and vacancies, with experiments revealing the existence of a denuded zone in the vicinity of interface regions. ${ }^{8,9}$ Thus the nc structure is expected to have increased radiation resistance due to its high density of GB's and with grain sizes that are comparable to the maximum cascade volumes.

Recent MD simulations of cascades in nc Ni have confirmed that the GB does indeed attract SIA's early in the cascade evolution via the replacement collision sequence (RCS) mechanism. ${ }^{6}$ This influences the vacancy/interstitial recombination rate, leaving behind a vacancy rich defect structure in the grain. The primary damage state in $\mathrm{nc} \mathrm{Ni}$ therefore differs from the single-crystal cascade state; for example, stacking fault tetrahedra are more frequently observed in the nc samples and they are usually larger in size. ${ }^{5}$ At a later stage of development of the primary damage, the SIA's that did not end up in the GB's via RCS's form clusters and enter a thermal phase of activity. In a crowdion configuration, these SIA clusters are seen to move via onedimensional/three-dimensional (1D/3D) motion to GB's, to regions containing GB dislocations and triple junctions, finally annihilating with free volume therein. ${ }^{7}$ Such $1 \mathrm{D} / 3 \mathrm{D}$ diffusion activity has been described via a low thermal migration barrier ${ }^{10-12}$ and more recently via dissipative Brownian motion. ${ }^{13}$
Former studies on single crystals demonstrated that SIA clusters containing more than three SIA's move only via 1D movement. ${ }^{1}$ 1D glide of clusters in Fe described by their center of mass has been described as the result of almost independent jumps of individual crowdions. ${ }^{14}$ This model, however, is unable to describe the crossover of individually moving SIA's to that of SIA's in a dislocation loop. More recently, the movement of smaller and larger sized SIA clusters has been described in terms of the soliton solution of the sine-Gordon equation and by the many-crowdion FrenkelKontorova model, respectively. ${ }^{15}$ In the work of Dudarev, the crossover from a small cluster to a dislocation loop occurs for clusters containing more than three SIA's where an inequivalence of crowdion widths is seen within the larger cluster. Recently we observed the movement of a six-SIA crowdion cluster formed during a $5-\mathrm{keV}$ primary knock-on atom (PKA) cascade in a nc structure, via $1 \mathrm{D} / 3 \mathrm{D}$ motion along one of its crowdion axes, changing its direction close to the GB to move along another close-packed plane before being absorbed by the GB. ${ }^{7}$

In this paper, the origin of the $1 \mathrm{D} / 3 \mathrm{D}$ motion of large clusters in nc displacement cascades is studied. This is achieved by superimposing the SIA activity resulting from irradiation over the local pressure variation prior to irradiation. It is shown that initially the SIA cluster sees the GB as a homogenous two-dimensional region of tensile pressure, which in the present work we refer to as a "defect collector plate." 16 However when the cluster approaches the GB, it sees the GB as a detailed structure with compressive and tensile regions, and correspondingly adapts its direction of motion to arrive at an area of localized tensile pressure.

\section{SAMPLE PREPARATION AND SIMULATION TECHNIQUE}

A nc Ni sample is synthesized using a Voronoi construction with random nucleation of seeds and random crystallographic orientation ${ }^{18}$ resulting in a fully $3 \mathrm{D}$-grain network 
structure with general GB's with all kinds of misorientation. The sample contains 1.2 million atoms and is relaxed to a minimum enthalpy at $300 \mathrm{~K}$. The PKA's are introduced in a central grain by imparting the energy and velocity onto one atom and allowing the sample to evolve via MD with the dissipation of energy through the system. Heat is removed at the periodic boundaries in an outer cubic region by rescaling of the atomic velocity.

To adequately represent the displacement cascade, the second moment tight-binding potential of Cleri and Rosato ${ }^{19}$ is employed with a modification in the short-range form according to Ziegler $e t a l{ }^{20}$ This more accurately describes the high-energy collision dynamics of the early ballistic phase.

A medium-range topological bond analysis technique ${ }^{21}$ is employed to visualize the atomic structure of the displacement cascade and the GB's present in the sample. By allocating a color code scheme to the local crystalline symmetry of each atom, various structures are easily distinguishable. This color code scheme comprises fcc atoms defined by gray, 12-coordinated non-fcc atoms by green, non 12-coordinated atoms by blue, and first nearest-neighbor hcp coordinated atoms by red spheres.

To investigate the spatial dependence of the pressure field within the nc structures, we consider first the global hydrostatic pressure for a volume $V,{ }^{22}$

$$
P=\left\langle\frac{1}{3 V} \sum_{i}\left(m v_{i}^{2}+\frac{1}{2} \sum_{\langle j\rangle} \vec{F}\left(r_{i j}\right) \cdot \vec{r}_{i j}\right)\right\rangle .
$$

Here the summations are over all $N$ atoms within the volume $V$ and $\langle\cdot\rangle$ represents an ensemble average or equivalently a long-time average. In the thermodynamic limit of large $V$, this represents the true bulk homogeneous hydrostatic pressure. To obtain an estimate of the spatial pressure variation one may write an expression for the local atomic pressure for atom $i$ :

$$
P_{i}=\left\langle\frac{1}{3 \Omega}\left(m v_{i}^{2}+\frac{1}{2} \sum_{\langle j\rangle} \vec{F}\left(r_{i j}\right) \cdot \vec{r}_{i j}\right)\right\rangle,
$$

where $\Omega$ is the representative volume for atom $i$. It is however generally known that for such a volumetric partition of the pressure, Eq. (1) lacks momentum conservation across its corresponding surfaces ${ }^{23}$ and as the volume approaches the atomic scale [as in Eq. (2)] this leads to non-negligible artifacts such as oscillatory behavior in strongly inhomogeneous systems.

More recently, a systematic approach has been developed that can more accurately represent local pressure ${ }^{24}$ via

$$
P_{\Omega}=\frac{1}{3 \Omega}\left\langle\sum_{i}\left(m v_{i}^{2} \Lambda_{i}+\frac{1}{2} \sum_{\langle j\rangle} \vec{F}\left(r_{i j}\right) \cdot \vec{r}_{i j} l_{i j}\right)\right\rangle,
$$

where $\Omega$ is now the volume of some region of interest. In Eq. (3), $\Lambda_{i}$ is unity if atom $i$ is within the volume element and zero otherwise, and $l_{i j}$ is the fraction of the length of the bond between atoms $i$ and $j$ that lies within the volume element. Equation (3) rigorously satisfies conservation of linear momentum for the chosen volume. In the present work we choose the volume element to be a sphere centered on each atom, and define the resulting pressure of that sphere as the local pressure of the central atom. The radius of the sphere is taken as $4 \AA$ and contains $\approx 19$ atoms.

Such a choice of spatial element is not a volume conserving partition of the global volume and therefore the summation of the individual pressures from Eq. (3) will not add up to the global pressure of the system (which is on average zero). Moreover, for the more homogeneous regions within the grains, the local volume pressure does not converge to the correct thermodynamic grain pressure. This can be remedied by choosing a larger volume element for each atom resulting in a reduction of spatial pressure resolution. Equation (2) on the other hand sums correctly to the global zero pressure value and also the local atomic pressure within the grain converges to the true thermodynamic grain pressure. As already mentioned it however suffers from unphysical oscillations for strongly inhomogeneous environments. In the present work we employ both methods to investigate the internal pressure distribution of the nc system, using Eq. (3) for investigation of the local GB region and Eq. (2) for investigation of the asymptotic pressure behavior within a grain due to a GB.

For both methods a time average is required in order to average over thermal vibrations. The duration of such a time average must be of $\sim 1 \mathrm{ps}$, which is typically several atomic vibrational periods. It is for this reason that the pressure prior to irradiation is used since such time averages cannot be performed during primary cascade evolution. For visualization, the color scheme of the pressure $p$ ranges from $-1.2 \mathrm{GPa}$ (purple) to $2.8 \mathrm{GPa}$ (red), where a positive value represents compression and a negative value represents tension. This range was chosen for optimal contrast since the average grain pressure using Eq. (3) was $0.8 \mathrm{GPa}$.

\section{RESULTS AND DISCUSSION}

\section{A. The GB as a defect collector plate}

Figure 1 shows a section of the Ni sample before the introduction of the PKA. In Fig. 1(a) the atoms are colored by their crystallinity, and in Fig. 1(b) the atoms are colored according to the local average pressure derived from Eq. (3). When looking at GB's in terms of crystallinity, the GB and triple junction (TJ) regions are rather narrow containing a maximum of 3 or 4 atomic layers. When the same sections are however examined in terms of local pressure the following observations can be made: (1) the GB's are made up of adjacent regions of high compression and tension compared to the pressure present in the grain interior; (2) some of the GB's have a compressive side towards one grain and a tensile side towards the other grain; (3) the GB width and the size of the triple junctions are larger when defined in terms of pressure with some of the compressive and tensile regions in the GB extending many atomic layers into the grain, and (4) the grain interior shows slight variations in local pressure.

Defining the GB as non-fcc atoms (16.2\% of atoms in the sample), the overall GB pressure using Eq. (3) averages to a tensile pressure, whereas the grain interior sums to a compressive pressure of $0.8 \mathrm{GPa}$. When using Eq. (2) the same trend is seen, ${ }^{25}$ however the average pressure of the grain 


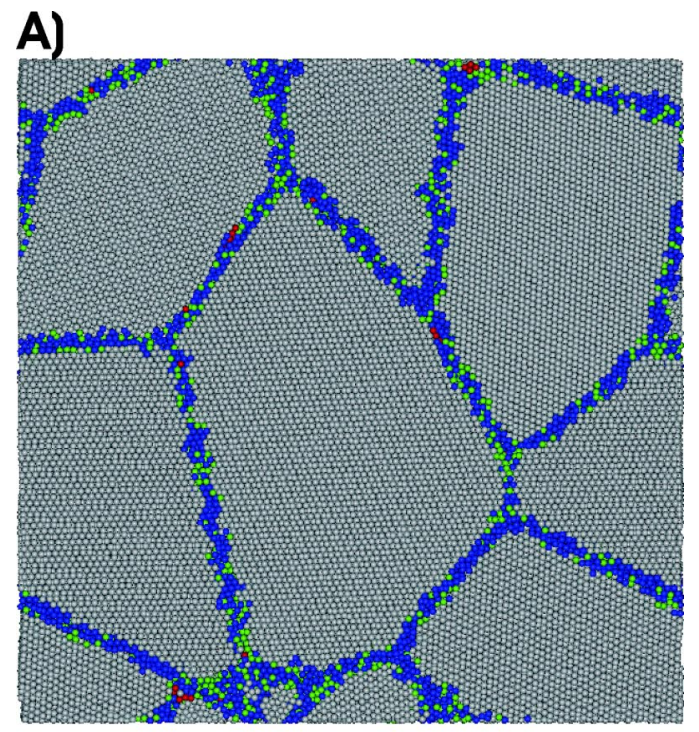

B)

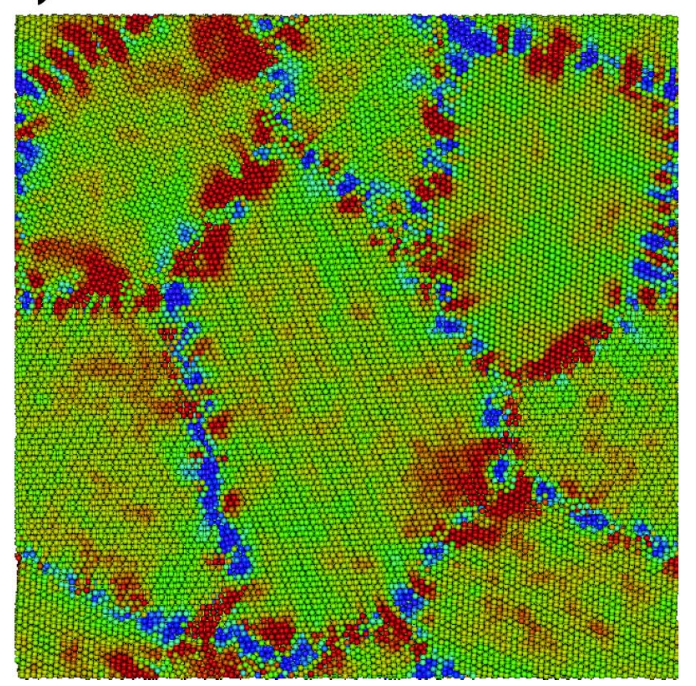

FIG. 1. (Color online) A section of the 3D sample showing (a) the crystalline order of atoms and (b) the local hydrostatic pressure associated with each atom.

interior now sums to the correct value of about $80 \mathrm{MPa}$. As already discussed in the preceding section this difference between Eqs. (2) and (3) is an artifact of the chosen small atomic volume of radius $4 \AA$. Since the GB regions contain strong variations between the tensile and compressive pressures that can extend into the grain interior, a second estimate of the GB and grain interior was undertaken. Summation of the pressure in a broader interphase region encompassing fcc atoms close to non-fcc atoms (containing now $31.8 \%$ of atoms in the sample) was found to also produce an overall tensile pressure in the GB regions and once again compressive pressure within the reduced grain interior.

The stress away from the GB's decreases rapidly, although not very homogenously. For boundaries made of simple dislocation arrays and satisfying Frank's equation, the decrease away from the GB's can be described by an exponential decay $\exp (-2 \pi x / D)$ when $x \gg D / 2 \pi{ }^{28,27}$ Here $x$ is the distance to the GB dislocation and $D$ is the distance be-

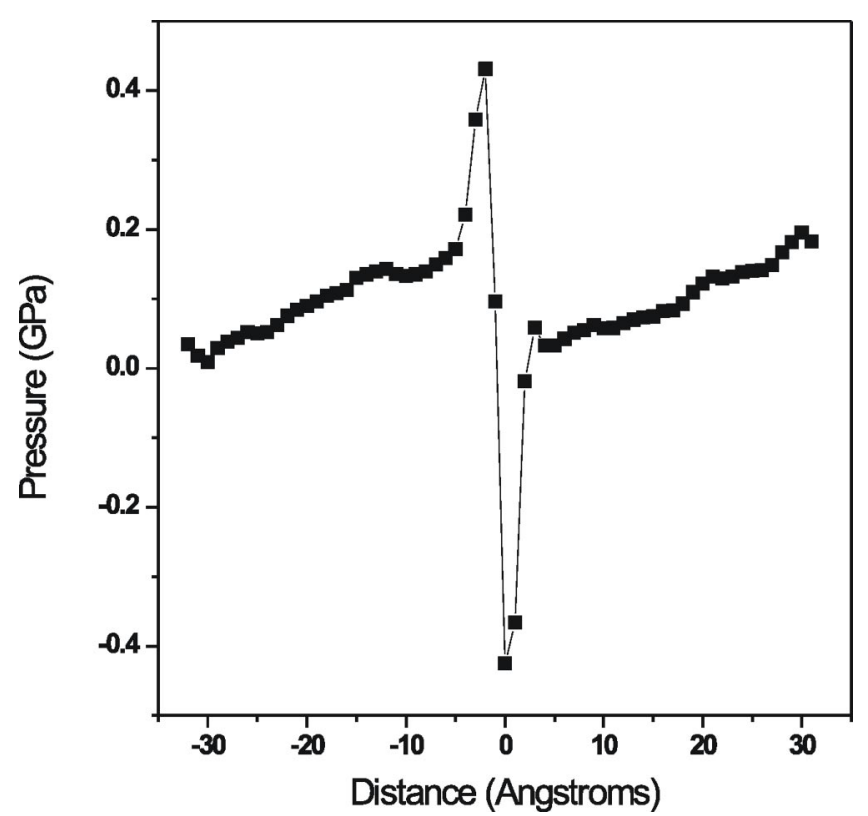

FIG. 2. The behavior of the asymptotic pressure of a section of the sample in the vicinity of grain boundary 1-5. The average pressure in $4 \AA$ thick slices at $0.2 \AA$ intervals is shown as a variation of distance from the grain boundary.

tween GB dislocations. We have estimated the distance between GB dislocations for GB's in which SIA clusters are seen to move towards in our 12-nm grain sized nc sample using two methods: by counting the number of extra planes in one grain compared with the neighboring grain using the crystallinity of the atoms, and by counting the number of regions of similar pressure and the frequency of fluctuation. This measure was performed in two almost perpendicular directions of the GB in order to account for the 3D GB structure and then averaged. For GB 13-9 (Figs. 3 and 4) $D=15.0 \AA$, with an average of 9 dislocations in the length of the GB. GB 1-5 (Fig. 5) contains 14 dislocations along the GB with an average distance of $13 \AA$. The values obtained for $D / 2 \pi$ are very small, $2.38 \AA$ for GB $13-9$ and $2.05 \AA$ for GB 1-5, which would result in a very fast drop of the stress away from the GB's. However due to the Voronoi construction technique and the inherent time scale restriction of MD, the GB's in computer generated nc samples are expected to be general GB's having a nonequilibrium structure that may not fulfil Frank's equation. Therefore, the stress fields of the GB dislocations will not cancel each other so effectively and long-range stresses in the grain interior might be expected. Moreover, due to the small grain size, the average pressure within the grain will be a result of other nearby GB's, resulting in the observed average compressive pressure within the grain and an average tensile pressure within the GB region.

Figure 2 displays the average pressure as a function of distance to the GB 1-5 (Fig. 5). For the calculation of the average pressure, a large pill box section of thickness $4 \AA$, perpendicular to GB 1-5 was taken and the average pressure was calculated using Eq. (2) in steps of $0.2 \AA$ from the GB. The horizontal distance scale is set so that GB 1-5 is situated at zero, the negative values represent the perpendicular distance away from the GB in grain 5 and the positive values 


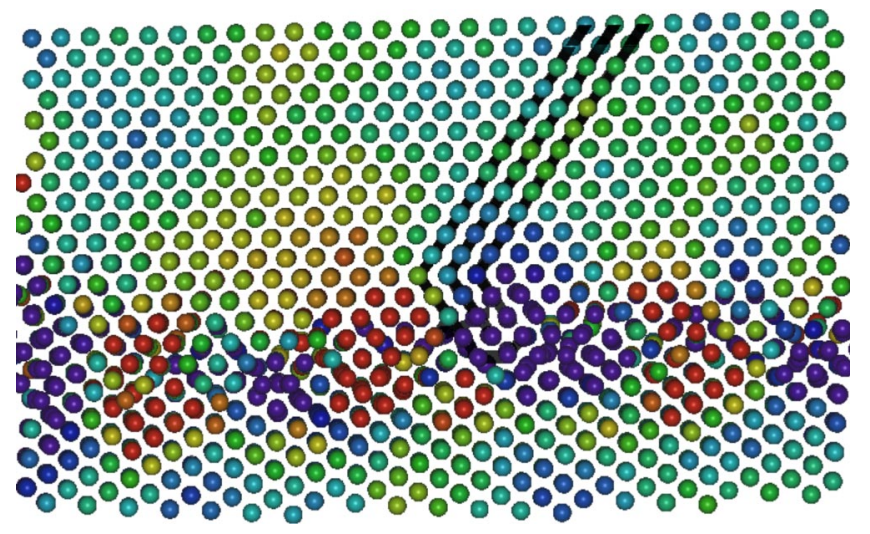

FIG. 3. (Color online) Close up of the local pressure associated with the grain boundary 13-9 region. Black lines indicate the $1 \mathrm{D} / 3 \mathrm{D}$ movement of a 6-SIA cluster to the grain boundary region.

represent the corresponding distance in grain 1 . The pressure anomaly at the original is a clear signature of the GB, the integration of which yields a net tensile pressure. The predominant effect of the GB extends no more than a nanometer into the grain, with an effective "tail" of pressure variation extending deep into the grain. We note that such a variation cannot be due only to GB 1-5, but also other nearby GB's where for instance the GB's directly opposite to GB 1-5 (on either side) are a distance of about 8-9 nm away. Thus the effect of the GB's can be felt throughout the grain and a SIA produced by a cascade in the center of a grain will experience the GB as a "defect collector plate." The effective attraction of SIA's to the GB is therefore expected to be dependent on the distance between SIA's and all surrounding GB's. An important observation for GB 1-5 is that away from the GB region the sign of the pressure gradient differs depending on from which grain, GB 1-5 is approached, whereas closer to the GB, it is the sign of the pressure profile that is different. Thus both the short-range and long-range properties of this GB in terms of a SIA sink would differ depending on which grain the SIA's are located in.

\section{B. Clusters moving in stress gradients}

In what follows, the reader is reminded that the pressure shown details the structure of the GB before the cascade has been introduced in order to show the regions of attractions without the added complications of thermal noise and heat of a cascade which would result in a much less clear result of the pressure gradients present due to the 1-ps framework that the pressure average is calculated over. As such, it is important to note that visualization done in this way does not consider the image forces of the SIA clusters on the GB's during the displacement cascade. As a first approximation, this may be done because of the relatively small force that a SIA cluster has on a GB. ${ }^{17}$

During a 5-keV cascade simulation in the $\mathrm{Ni} 12 \mathrm{~nm}$, a 6-SIA crowdion cluster is formed in the irradiated grain as the cascade core region cools. ${ }^{7}$ Figure 3 shows the movement of the 6-SIA cluster (the black lines indicating the displacement of atoms during the cascade evolution) moving

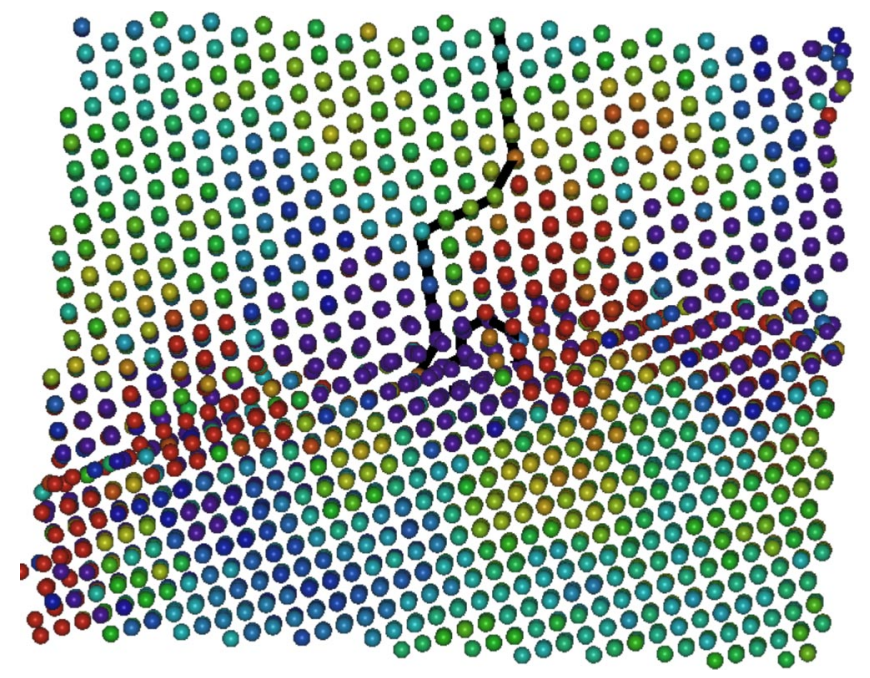

FIG. 4. (Color online) Local pressure of grain boundary 13-9 region as a single SIA moves via 1D/3D motion, avoiding high compressive (red) pressure as it moves to the grain boundary.

along its crowdion axis, in a close-packed direction. The atoms in this figure are colored according to the local atomic pressure of Eq. (3). The high compressive/tensile (red/ purple) pressure regions in the GB are clearly distinguishable. The cluster moves 1D towards the GB until it has a 6.7 $\AA$ (approximately two lattice constants) separation from the GB and then changes its direction. If the cluster were to continue 1D along its path, it would reach the GB at a region of high compressive pressure. That the 6-SIA cluster does not choose from the beginning a path that will allow it to move 1D along a close-packed plane reaching a region of high tensile pressure within the GB can be explained for example by its inability to distinguish between far off regions of pressure so that the cluster may be thought of as being "short sighted." In other words the cluster senses the far away interphase region as a homogeneous slab of undistinguishable tension, in a sense a "defect collector plate" with overall tensile pressure, and cannot feel the detailed compressive/tensile pressure fields of the GB. ${ }^{16}$ The SIA cluster therefore starts its journey along a close-packed plane to the interphase region attracted by the overall tensile pressure, a direction that later on might have to be changed to another close-packed direction when the original path leads to a compressive region at the GB. In single crystals, where this type of strong modulation between compressive and tensile regions does not exist, clusters of four or more SIA's have always been observed to move along $1 \mathrm{D}$ paths. ${ }^{1}$

The six-SIA cluster shown in Fig. 3 enters the GB at a GB dislocation where excess free volume exists. After annihilation of the SIA in the GB and further relaxation, the final structure of the GB remains similar to the initial structure, with a local reorganization of the GB to accommodate the incoming SIA's. ${ }^{7}$

A similar event is shown in Fig. 4 where a mono-SIA, produced in the same $5-\mathrm{keV}$ cascade, moves along a $1 \mathrm{D} / 3 \mathrm{D}$ path. Again, the SIA senses the GB first as a region of uniform tensile pressure until it is close enough to experience 

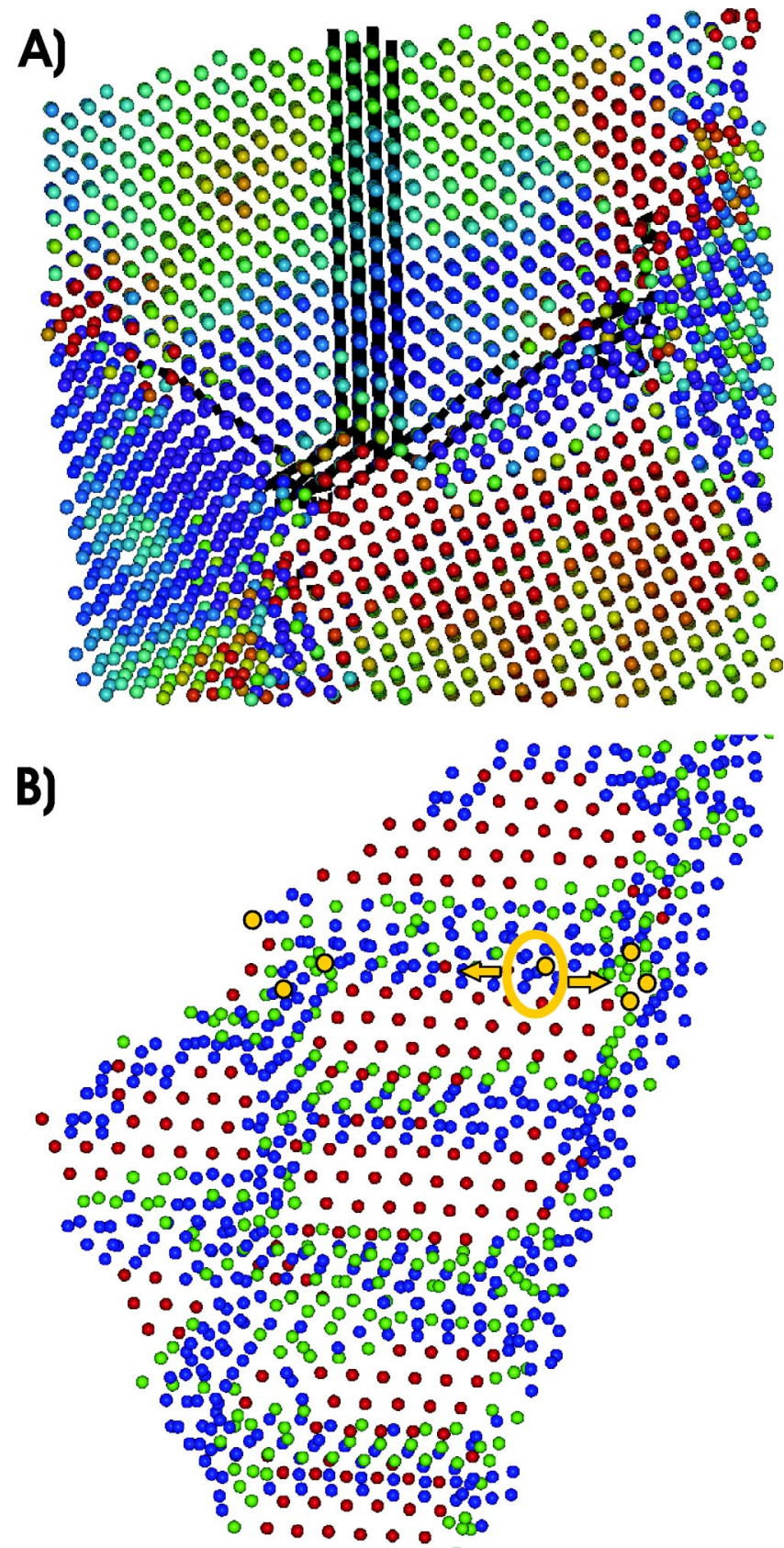

FIG. 5. (Color online) Movement of a 7-SIA cluster (black lines) to the grain boundary and triple junction regions. (b) Perpendicular view of the grain boundary plane indicating the entrance region of the 7-SIA cluster (yellow outline); the direction of the SIA's in the grain boundary (yellow arrows); where SIA's end up (full yellow circles).

the detailed pressure variations of the GB. In this case the SIA changes its direction several times to avoid the region of high compressive pressure and moves away from the local high compressive pressure, directing itself towards a region of high tensile pressure, finally traveling in the GB until it finds free volume to annihilate with.

Figure 5 shows a 7-SIA cluster resulting from a $10-\mathrm{keV}$ cascade in the nc-Ni sample. The SIA cluster moves towards
GB 1-5 that is close to a $\Sigma=3$ structure. The boundary shows a tensile area on one side (the side where the cluster is arriving) and a compressive area on the other side (see Fig. 2 ). When the SIA arrives close to the GB, it sees a region with tensile pressure but there is no free volume available for annihilation. Some of the SIA's are moving within the GB and the others are still in the grain when this change in direction occurs. This GB contains a significant degree of crystalline order ${ }^{26}$ with the GB being made up of portions of (111) twin boundary planes (red atoms) separated by nonfcc/non-hcp regions (blue/green atoms) forming a step between the nearby twin planes as shown in Fig. 5(b). The circled area shows where the large SIA cluster enters the GB and the direction of SIA movement is indicated by the arrows. The yellow circles indicate the final position of the SIA's. The lack of free volume in this highly structured GB allows only one SIA to annihilate in the GB, the others moving along the step region to nearby TJ's. The interphase region of the irradiated grain contains a highly tensile region, whereas the interphase region on the other side of the GB is highly compressive. On entrance to the GB, the SIA cluster senses a large wall of high compression before it and the SIA's disperse within the GB. Experimentally highly structured step GB's have been observed to create/destroy defects only at interfacial dislocation sites. ${ }^{28}$ That this GB attracts the large SIA cluster, although usually not a strong sink source, strengthens the idea that the SIA cluster senses the interphase region as a defect collector plate, a region of indistinguishable high tensile pressure rather than that of the local highly fluctuating pressure regions actually present.

\section{CONCLUSION}

The above analysis has shown that general grain boundaries in nanocrystalline structures contain alternatively regions of high compression and high dilatation. When selfinterstitial-atom clusters are formed within a cascade they first experience the GB as a uniform defect collector plate with tensile pressure and are therefore attracted by the grain boundary, moving along a single close-packed direction. However when the cluster arrives within a few atomic layers of the grain boundary, the cluster sees the strong pressure gradients in the grain boundary and changes its direction in order to arrive at a region of tensile pressure, to finally annihilate with free volume available in the grain boundary. The pressure gradient influence is sufficient to affect the direction of self-interstitial clusters containing up to six selfinterstitial atoms, undergoing $1 \mathrm{D} / 3 \mathrm{D}$ motion in order to maintain a path along low-pressure gradients in their movement to the grain boundary.

\section{ACKNOWLEDGMENTS}

The authors would like to thank A.P. Sutton for the discussions and concept of the GB as a homogeneous "defect collector plate." M.S. acknowledges the financial support of EFDA (Grant No. TW4-TTMS-007) and the BBW (Grant No. 98.0098). M.V. acknowledges the financial support of the Swiss-FN (Grant No. 20/61837.00-1). 
${ }^{1}$ D.J. Bacon, F. Gao, and Yu.N. Osetsky, J. Nucl. Mater. 716, 1 (2000)

${ }^{2}$ F.J. Perez-Perez and R. Smith, Nucl. Instrum. Methods Phys. Res. B 153, 136 (1999).

${ }^{3}$ F.J. Perez-Perez and R. Smith, Nucl. Instrum. Methods Phys. Res. B 164-165, 487 (2000).

${ }^{4}$ M. Samaras, P.M. Derlet, H. Van Swygenhoven, and M. Victoria, M. Phil Mag A (to be published).

${ }^{5}$ M. Samaras, P.M. Derlet, H. Van Swygenhoven, and M. Victoria, Nucl. Instrum. Methods Phys. Res. B 202, 51 (2002).

${ }^{6}$ M. Samaras, P.M. Derlet, H. Van Swygenhoven, and M. Victoria, Phys. Rev. Lett. 88, 125505 (2002).

${ }^{7}$ M. Samaras, P.M. Derlet, H. Van Swygenhoven, and M. Victoria (unpublished).

${ }^{8}$ M. Rose, A.G. Balogh, and H. Hahn, Nucl. Instrum. Methods Phys. Res. B 127/128, 119 (1997).

${ }^{9}$ Y. Chimi, A. Iwase, N. Ishikawa, M. Kobiyama, T. Inami, and S. Okuda, J. Nucl. Mater. 297, 355 (2001).

${ }^{10}$ Yu.N. Osetsky, M. Victoria, A. Serra, S.I. Golubov, and V. Priego, J. Nucl. Mater. 251, 34 (1997).

${ }^{11}$ T. Diaz de la Rubia and M. Guinan, Phys. Rev. Lett. 66, 2766 (1991).

${ }^{12}$ D.J. Bacon and T. Diaz de la Rubia, J. Nucl. Mater. 216, 275 (1994).

${ }^{13}$ S.L. Dudarev, Phys. Rev. B 65, 224105 (2002).
${ }^{14}$ A.V. Barashev, Yu.N. Osetsky, and D. Bacon, Philos. Mag. A 80, 2709 (2000)

${ }^{15}$ S.L. Dudarev, Philos. Mag. 83, 3577 (2003).

${ }^{16}$ A.P. Sutton (private communication).

${ }^{17}$ X.H. Liu, F.M. Ross, and K.W. Schwarz, Mater. Res. Soc. Symp. Proc. 673, 4.2 (2001).

${ }^{18}$ G.Z. Voronoi, J. Reine Agnew. Math. 1 34, 199 (1908).

${ }^{19}$ F. Cleri and V. Rosato, Phys. Rev. B 48, 22 (1993).

${ }^{20}$ J. Ziegler, J.P. Biersack, and U. Littmark, The Stopping Range Of Ions in Solids (Pergamon, New York, 1987).

${ }^{21}$ D.J. Honeycutt and H.C. Andersen, J. Phys. Chem. 91, 4950 (1987).

${ }^{22}$ D. Frenkel and B. Smit, Understanding Molecular Simulation: From Algorithms to Applications (Academic, San Diego, CA, 2002).

${ }^{23}$ J.F. Lutsko, J. Appl. Phys. 64, 1152 (1988).

${ }^{24}$ J. Cormier, J.M. Rickman, and T.J. Delph, J. Appl. Phys. 89, 99 (2001).

${ }^{25}$ A. Hasnaoui, H. Van Swygenhoven, and P.M. Derlet, Acta Mater. 50, 3927 (2002).

${ }^{26}$ H. Van Swygenhoven, D. Farkas, and C. Caro, Phys. Rev. B 62, 831 (2000).

${ }^{27}$ D. Hull and D.J. Bacon, Introduction to Dislocations, (Butterworth-Heinemann, Oxford, 2001).

${ }^{28}$ A.P. Sutton and R.W. Ballufi, Interfaces in Crystalline Materials (Clarendon, Oxford, 1995). 